SSC10-I-3

\title{
The CubeSat Heliospheric Imaging Experiment (CHIME)
}

\author{
Craig DeForest \\ Southwest Research Institute \\ 1050 Walnut Street, Suite 300, Boulder, CO 80302; 303-546-6020 \\ craig.deforest@swri.org
}

\author{
John Dickinson, Juan Aguayo, John Andrews, Michael Epperly, Tim Howard, Joe Peterson, Jennifer \\ Alvarez, Michael Pilcher \\ Southwest Research Institute \\ 6220 Culebra Rd, San Antonio, TX 78238; 210-522-5826 \\ john.dickinson@swri.edu \\ Craig Kief, Steve Suddarth \\ Configurable Space Microsystems Innovations \& Applications Center \\ 2350 Alamo Ave SE, Suite 100, Albuquerque, NM 87106; 505-242-0339 \\ craig.kief@cosmiac.org \\ James Tappin \\ National Solar Observatory \\ PO Box 62, Sunspot, NM, 88349; 575-434-7023 \\ jtappin@nso.edu
}

\begin{abstract}
We describe a CubeSat mission to predict and diagnose space weather events at Earth by tracking the interplanetary disturbances that cause those effects. Our demonstration mission, the CubeSat Heliospheric Imaging Experiment (CHIME), is a wide-field sky camera that can image large, tenuous clouds of material as they cross the inner solar system en-route to Earth. These clouds, such as interplanetary coronal mass ejections (ICMEs), are produced by magnetic activity on the surface of the Sun, and consist of billions of tons of magnetized plasma that streak across the solar system at up to 8 million $\mathrm{km} /$ hour. Impact of ICMEs on the Earth's magnetosphere is the main cause of magnetic storms, aurora, ionospheric radio interference, intermittent satellite radiation exposure, and related space weather activity at Earth.
\end{abstract}

ICME tracking requires modest resolution and data rates, and is well suited to the CubeSat platform. CHIME will enable ongoing developmental space weather prediction, demonstrate the heliospheric imaging concept on the CubeSat platform, and advance the state of CubeSat readiness for many applications. Further, CHIME is a stepping stone to an agile, operational space weather imaging system, using moderate numbers of extremely inexpensive, redundant spacecraft to achieve robust operational reliability from commercial grade parts.

\section{INTRODUCTION}

Recent scientific results show that operational heliospheric imaging can greatly enhance both predictive and current knowledge of space weather conditions. CHIME explores Earth's near-space environment by tracking solar-originated interplanetary events and associating them with their terrestrial effects. Understanding magnetic storms from terrestrial data alone is infeasible, because the root cause lies in solar effects. Further, ICMEs evolve and interact with the interplanetary medium as they propagate, so that making accurate predictions is difficult from solar data alone. Heliospheric imaging uniquely allows reliable prediction (with approximately one day lead time and better than two hour uncertainty) of the time and strength of the magnetic storms that modify the ionosphere and affect radio communications, GPS, and other space assets.

CHIME is a demonstration heliospheric imager that prototypes an operational constellation to predict space weather and inform understanding of related ionospheric effects as they happen, augmenting the terrestrial space environment monitoring capabilities in existence. CHIME fills an urgent operational need: despite the demonstration that heliospheric imaging 
greatly improves prediction and understanding of space weather effects, all current heliospheric imagers will become useless for terrestrial space weather work as early as mid 2011. We are currently experiencing an unexpectedly long and deep period of solar minimum activity, but the Sun is showing signs of new-solarcycle activity and ICME activity is expected to become more active beginning in the 2011-2012 time frame. Deploying the CHIME constellation will help fill the impending void in Heliospheric imaging and magnetic storm prediction in an affordable and effective way.

\section{HELIOSPHERIC IMAGING AND SPACE WEATHER}

"Space Weather" describes a large suite of effects, including the variations in total electron content and excitation of Earth's ionosphere and magnetosphere. These quantities are primarily dependent on energy injection from variations in the solar wind and other solar-originated effects and cannot be well understood without reference to the solar events that cause them.

The most severe space weather effects, geomagnetic storms, are large magnetic disturbances that energize the van Allen belts, cause aurora, shift the magnetopause, and give rise to energetic particle showers. They have been associated with power station failure, spacecraft damage and destruction, increased radiation dosage for airline flyers and astronauts, and disruption of radio communications. ${ }^{1}$ Geomagnetic storms are caused by the impact of clouds of material, called Interplanetary Coronal Mass Ejections (ICMEs), that originate from the Sun.

ICMEs (also called "CMEs" in the context of solar physics) are large, fast-moving clouds of solar plasma that has been ejected into interplanetary space.
Typically containing masses on the order of $10^{12} \mathrm{~kg}$ and spanning tens of degrees in solar latitude [e.g. 2], they are the largest eruptions of matter and energy from the Sun. Near the Sun, CMEs have traditionally been observed with white light coronagraphs, which block out the majority of light from the Sun to reveal the faint surrounding corona.

Far from the Sun, ICMEs have most frequently been detected by in situ probes, which traverse the interplanetary medium and detect ICMEs directly via local effects such as the magnetic field and particle streams as the ICME sweeps over the spacecraft. Such spacecraft only provide a single-track view of the ICME. Further, predictive capability depends on distance from Earth, and warning given by the current aging upstream monitor (ACE, at the Earth-Sun L-1 point) can be as short as 15-20 minutes for the fastest moving events, insufficient for tactical planning.

A relatively new field, "heliospheric imaging," has predictive power that is far more accurate than extrapolation from near-Sun measurements, yields far longer lead times than L-1 in situ monitors, and gives far better understanding of the structure and evolution of the ICMEs that cause space weather than either of those technologies.

\section{Heliospheric Imagers}

ICMEs are visible against the blackness of space as faint diffuse glowing structures due to scattering of sunlight by the electrons in the cloud, and they can be tracked across the inner solar system with carefully baffled wide-field cameras. The first ICME glow data were collected in the 1970 s by the E9 zodiacal light instrument on board the Helios space probe, which demonstrated that ICMEs could be detected at large
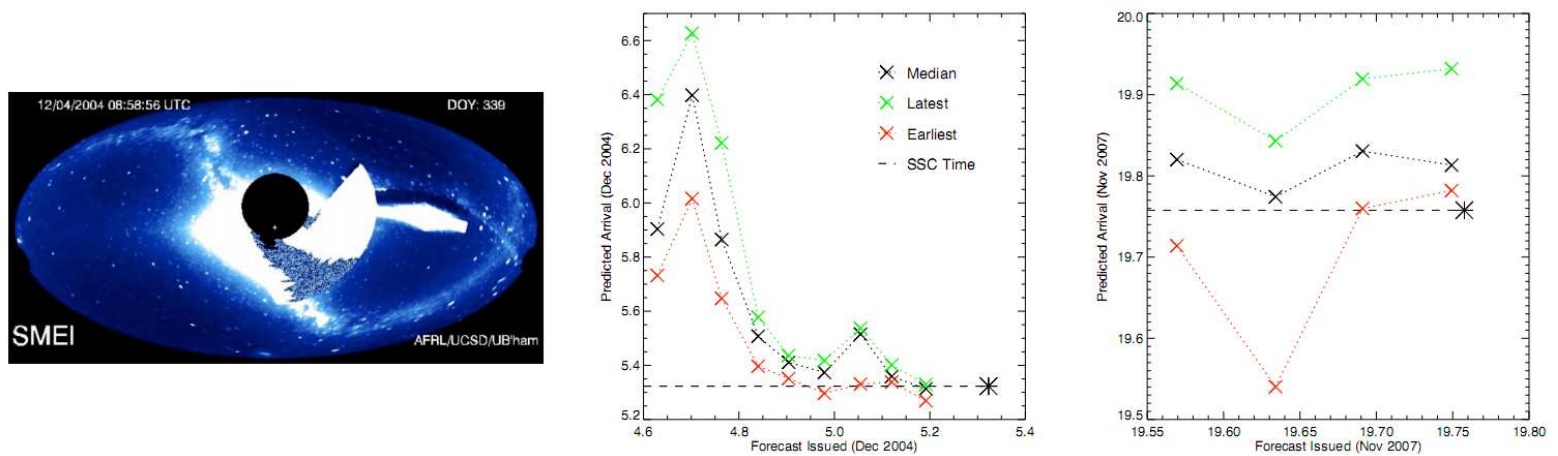

Figure 1: Heliospheric imaging (left) yields accurate space weather prediction. Mock prediction tests (including modeled system latencies) show \pm 2 hour precision more than a day in advance of the event (center), a tenfold improvement over current prediction techniques that do not use heliospheric imaging. Accuracy improves as the ICME approaches Earth (right). In addition to other science and technology development, CHIME demonstrates heliospheric imaging and event arrival prediction from a CubeSat. 
distances from the Sun. ${ }^{3}$ The first dedicated all-sky heliospheric imager, AFRL's Solar Mass Ejection Imager (SMEI), is on board AFRL's Coriolis spacecraft. ${ }^{4}$ SMEI continues to operate and has detected well over 300 ICMEs during its 7 year lifespan, leading to recent spectacular advances in the theory of space weather prediction. ${ }^{5}$ However, SMEI is nearing end-of-life and no replacement is currently underway. More recently, in 2006, NASA's twin STEREO deep-space probes included heliospheric imagers. They have detected ICMEs on their passage across the heliosphere. The STEREO HIs have further demonstrated the utility of heliospheric imaging, but a combination of limited field-of-view and exotic solar orbits will eliminate their ability to observe Earthdirected events by mid 2011.

Both STEREO-HI and SMEI will thus become unusable for terrestrial space weather monitoring, just as the solar cycle enters its rapid rise phase and ICME effects at Earth become important. The time is right to build a demonstration operational near-Earth heliospheric imager, to replace and augment the capabilities of SMEI and STEREO-HI before they are lost.

\section{Space Weather Forecasting: Current techniques}

The two most important effects driving magnetic storms are the ram pressure exerted on the magnetosphere by an impacting ICME, and the orientation of the ICME's internal magnetic field. Predicting the onset of the storm requires knowledge of the geometry and kinematics of the ICMEs as they leave the Sun and propagate across the inner solar system. The current state of the art for space weather prediction uses near-Sun empirical data, such as visual CME properties in coronagraph data from the SOHO/LASCO instrument, solar flare location on the surface of the Sun, and ICME-related radio bursts, yielding an approximate 1-day arrival window and a poor prediction of the strength of an impending geomagnetic storm.

All three predictors suffer difficulties, because the interplanetary magnetic field (IMF) and other solar wind phenomena such as corotating interaction regions (CIRs) between different wind domains and preexisting, slow-moving ICMEs affect the evolution and propagation of each ICME differently. Some ICMEs slow down after they leave the field of view of LASCO, presumably due to interaction with the slower-moving solar wind; others speed up for reasons that are not yet understood. ICMEs do not propagate in straight lines, but are deflected in a hard-to-predict way by the IMF. Hence, without monitoring each ICME as it propagates, it is impossible to predict reliably whether Earth will be hit by the leading edge, a flank, or not at all. The difficulty holds even if the ejection geometry at the Sun is known.

Improving understanding of these effects is a major focus of NASA's Living With a Star (LWS) program, which seeks to identify the physics connecting the Sun and the Earth. Nevertheless, most techniques have borne little fruit due to the difficulty of assessing the effects of the interplanetary medium on propagating ICMEs. Webb et al. [2009] recently summarized the accuracies of many current ICME arrival time prediction techniques. ${ }^{6}$ The most accurate predicted arrival time cited was $+/-11$ hours from the ISPM magnetohydrodynamic model, first reported in 1995 . $^{7}$ Recent years have seen little progress in improving ICME arrival time prediction accuracy using modeling and solar data.

\section{Forecasting with Heliospheric Imagery}

Two recent papers by CHIME team members ${ }^{8,9}$ develop and demonstrate a method, called the "TH model," of interpreting heliospheric data to extract models of ICME propagation and predict arrival time and event
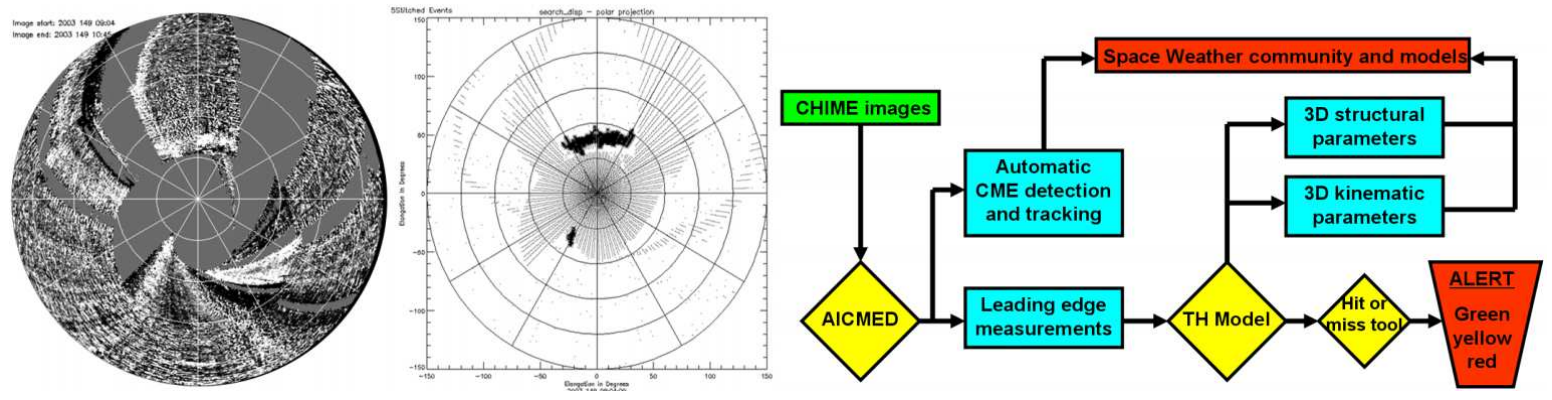

Figure 2: AICMED processes running-difference heliospheric images (left) to identify degraded data and ICME locus (center); together with CHIME, AICMED and TH would form a complete space weather warning system. 
strength at Earth. The method uses leading-edge determinations from time series of heliospheric images, and has been demonstrated using heliospheric imagery from SMEI. 8 conducted mock space weather forecasts on several events observed by SMEI; typical forecast performance is shown here in Figure 1. Predictions achieved +/- 2 hour precision more than a day in advance of the ICME impact, an order of magnitude improvement over non-heliospheric-imaging techniques.

Recent work by Tappin \& Howard has led to the development of AICMED, a code that can identify ICME locations automatically in heliospheric imaging data. A combination of CHIME, AICMED, and the TH model would form a complete end-to-end space weather warning system: CHIME data, automatically reduced and processed through AICMED, would yield automatic, accurate, reliable warnings of imminent space weather events one day in advance, with $+/-2$ hour precision in arrival time (Figure 2).

\section{LONG TERM VISION: CHIME CONSTELLATION ACHIEVES OPERATIONAL RELIABILITY}

CHIME is a pathfinder mission to raise TRL of imaging components and demonstrate heliospheric imaging within the CubeSat envelope. Once CHIME has flown successfully, it opens a new, low-cost, high-reliability pathway to operational space weather predictive imaging.

Many parties, including the Air Force and NOAA, have acknowledged the central importance of heliospheric imaging to space weather prediction at Earth, with its associated tactical and civil benefits. Building and billeting a single operational-class instrument has a lead time of order 10 years and a cost in the $\$ 100 \mathrm{M}$ range; CHIME can deliver similar results in under two years, with a much smaller system cost even for a full constellation.

The repeat construction lead time and construction total on-orbit cost are months and well under \$1M. CHIME CubeSats have minimal environmental requirements and can be cold-stored in ordinary briefcase-sized hard cases for rapid P-POD deployment as needed in the mass margin of practically any LEO-bound mission.

By flying a fleet of 3-5 separate autonomous CHIME CubeSats, none of which themselves have an operational reliability rating, it is possible to reduce the probability of system failure into the operational class.
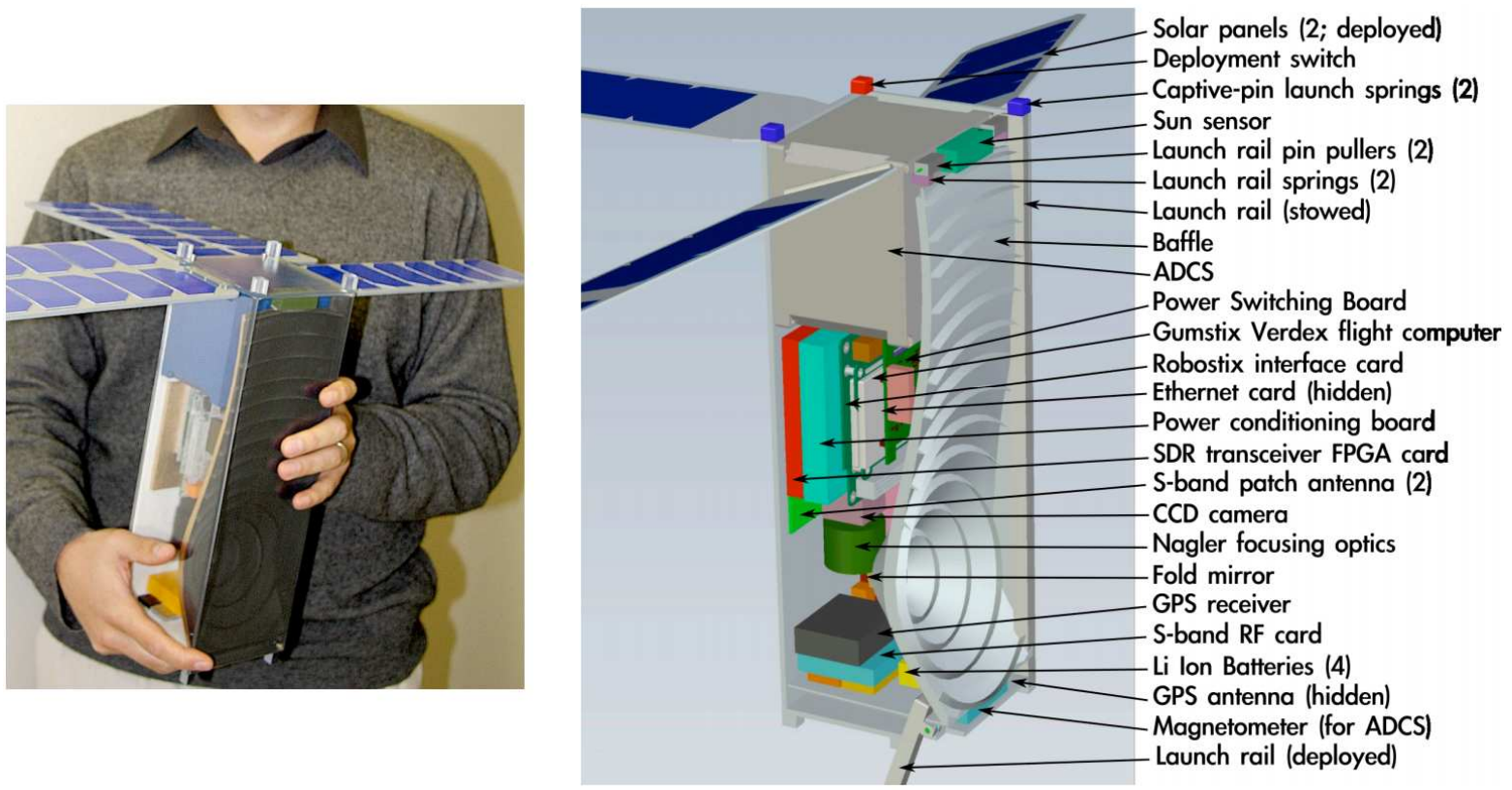

Figure 3: All CHIME systems fit within a 3U cubesat envelope. LEFT: 1:1 engineering model demonstrates layout and baffle geometry. RIGHT: recent render shows multiple design refinements. The entire $+Y$ face of CHIME is dominated by a large optical baffle to reduce incident sunlight by 13 orders of magnitude. The baffle is an integral part of the CHIME structure. The $+Y$ face launch rails deploy to the rear ( $-X$ rail shown stowed, $+X$ rail shown deployed) to eliminate glint. The lateral solar panel hinges are slanted 20 degrees to remove them from the baffle field of regard. 
Taking typical CubeSat lifetime to be 1 year, the worst case 10-year fleet cost is under $\$ 50 \mathrm{M}$. We expect that repetition with feedback on failure modes will yield further significant lifetime and cost benefits over the course of such a program. A 5 CubeSat fleet with a 1 year expected life for each CubeSat has a mean time between system failures of over 1,000 years (assuming a two month lead time to launch a spare CubeSat in case of single-spacecraft failure).

Given the very low repeat cost to deploy new CubeSats, extremely reliable fleet performance is thus possible at a fraction of the cost of a conventional operational-class mission.

\section{TECHNICAL DESCRIPTION}

CHIME will demonstrate operational space weather prediction using visible-light heliospheric imaging of ICME clouds as they cross the solar system. In a Sunsynchronous $400 \mathrm{~km}$ twilight orbit, a single CHIME spacecraft is capable of acquiring one maximal darksky map of the inner solar system per orbit, yielding a 90 minute cadence for ICME observations. This is sufficient to demonstrate ICME propagation and perform proof-of-concept space weather predictive studies, and matches the capabilities of the existing SMEI imager.

The CHIME spacecraft is a fully capable three-axis pointed vehicle with an imaging instrument, in a $3 \mathrm{U}$ CubeSat package (Figure 3). The spacecraft utilizes single-string design and primarily commercially available parts (Figure 6), and is intended to have a mission life of $\sim 1$ year and a repeat construction cost of well under $\$ 1 \mathrm{M}$ in quantity. Short design and assembly cycles and low launch cost yield a "pay as you go" operational capability: while any one CHIME spacecraft is useful primarily for demonstration of campaign science operations, a small constellation of CHIME satellites can provide higher observing cadence, shorter data latency, and operational level reliability for much lower cost than a traditional operational mission.

The CHIME instrument consists of a modest resolution (7-10 arcmin) visible light camera with a 60 degree field of view, mounted inside a dark baffle system that shields the camera aperture from the rays of the Sun and the Earth. The baffle system has a visible stray light rejection ratio of $10^{-13}$ in the solar direction and $10^{-9}$ in the anti-solar direction. The low-scatter imaging system further reduces stray light to a few $\times 10^{-15}$ near the center of the field of view, enabling clear imaging of the extremely faint ICME clouds that are themselves as faint as $10^{-14}$ of the solar surface brightness.
The baffle system occupies one entire "long" face of the spacecraft. The camera system is a long-exposure offthe-shelf scientific CCD camera with $1300 \times 1000$ pixel resolution and 16 bit photometric capability (with multiple combined exposures). Typical exposures are accumulated for 300 seconds as a series of shorter individual frames that are despiked and merged to make a clean "macro-exposure" image of the visible patch of the celestial sphere. The macro-exposure image is stored on-board for downlink.

The CHIME spacecraft is pointed using an off-the-shelf modular ADACS that is capable of arcsecond class pointing with an auxiliary sun sensor. The sun sensor consists of a $2 \mathrm{~mm}$ lens in front of a quad diode array. It defines the boresight of the spacecraft. The sun sensor is mounted on the sunward $(-Z)$ face and is hidden from the instrument by the first baffle vane. The flight computer is a general-purpose workstation operating at $300 \mathrm{MHz}$. Command and downlink are achieved via either an off-the-shelf packet radio in the UHF band or a custom software-defined radio card that is currently under development at SwRI using COSMIAC hardware. The CCD and flight computer are near the center of the spacecraft structure and surrounded by batteries and other bulky subsystems for maximal radiation shielding. Power is supplied by COTS deployable solar panels.

In normal operation, CHIME acquires images of a patch of sky with elongation angles between 35 and 95 degrees from the Sun and a roll angle that varies with phase of the CHIME orbit (near the current terrestrial zenith). This set of angles allows imaging of Earthdirected ICMEs from solar distances of approximately $0.5 \mathrm{AU}$ to $1.0 \mathrm{AU}$ (impact). Images from different orbital phases can be stitched together on the ground to form a mosaic covering all roll angles about the EarthSun axis. The two-dimensional image plane overcomes problems with aurora and radiation interference that were encountered with SMEI: each portion of the celestial sphere can be imaged from locations as far as $6000 \mathrm{~km}$ apart in the spacecraft's orbit, eliminating data "holes" from aurora and from particle showers in the polar caps and south Atlantic anomaly.

The images are despiked and compressed on board using the general-purpose CPU. CHIME can acquire a despiked, deep exposure image of the camera's field of view in under 5 minutes. In Sun-synchronous polar orbit, CHIME can acquire images throughout the orbit to deliver a complete map of the inner solar system once per orbit; imaging performance varies with phase of the year in non-synchronous polar orbits. A followon operational CHIME constellation would deliver several complete maps per 100 minute orbital period, 
and would not require Sun-synchronous placement for continuous monitoring.

\section{CHIME Baffle}

CHIME relies on both a multivane corral-type baffle and low-scatter focusing optics to reduce stray light to acceptable levels. Baffle technology is well understood. ${ }^{4}$ Between the baffle and low-scatter optics, the current CHIME design achieves better than $3 \times 10^{-}$ 15, yielding a generous margin in stray light performance.

To achieve deep stray light rejection, CHIME's baffle follows the STEREO/HI strategy of a circular corral with additional vanes in the solar direction. The baffle occupies an entire face of the $3 \mathrm{U}$ CubeSat form. Compared to STEREO/HI, CHIME's baffle is far smaller but also somewhat less capable, rejecting a nominal 13 orders of magnitude rather than 15 as in that mission. That is appropriate because CHIME's mission is to track strong, potentially geoeffective ICMEs rather than all disturbances in the heliosphere. Neglecting noise rejection due to CHIME's 2-D image plane, CHIME's stray light characteristics are comparable to those of SMEI, which has been proven capable of imaging ICMEs at brightness levels as low as a few $\times$ $10^{-16} B_{\text {sun }}$

The flight attitude program maintains the front $(-Z)$ face of the spacecraft pointing at the Sun, and rolls the baffle (on the $+Y$ face) to maintain it close to the terrestrial zenith. Multiple baffle vanes reduce the diffracted intensity by a factor of $\sim 15$ at each vane. The baffle structure is machined from a solid piece of T6061 aluminum and also functions structurally and as a radiative cooler. The structure is protected before and during launch by the P-POD capsule itself.
Several design features of the CHIME spacecraft further reduce glint and spacecraft-related stray light sources to enable high stray light rejection. For example, the lateral solar panels are tilted slightly away from the baffle face, to remove the bright leading face from the baffle's field of regard.

We have considered lunar stray light from the lateral direction. Preliminary analysis indicates that the baffle reduces stray moonlight to acceptable brightness levels when the Moon is more than 28 degrees outside the field of view. This reduces CHIME's range in azimuth from 360 degrees to slightly under 300 degrees during the half of the month when the Moon is in the field of view.

To better understand and characterize CubeSat-sized baffles, a portion of the CHIME mission is devoted to exploring the performance of the baffle with various off-points relative to the nominal position. In particular, we explore closer Sun-FOV angles than the nominal 30 degrees, testing the importance of the first few baffles encountered by the solar beam. Further, a 29 day 1-solar-rotation science period also demonstrates the lunar stray light performance for a complete lunar month.

\section{Optics \& Camera}

The focusing optics consist of a commercial wide-field achromatic telescope eyepiece made by TeleVue. The optical train has a focal length of $7 \mathrm{~mm}$ and an objective size of $5 \mathrm{~mm}$ for an f-ratio of 1.5 . The eyepiece system was selected for its physical robustness, compact size, low geometric distortion, high f-ratio, and excellent stray light properties. Radiation darkening of the glass and coatings is not a concern for the expected mission life and LEO environment. To fit within the CubeSat body and to enhance camera shielding from particle
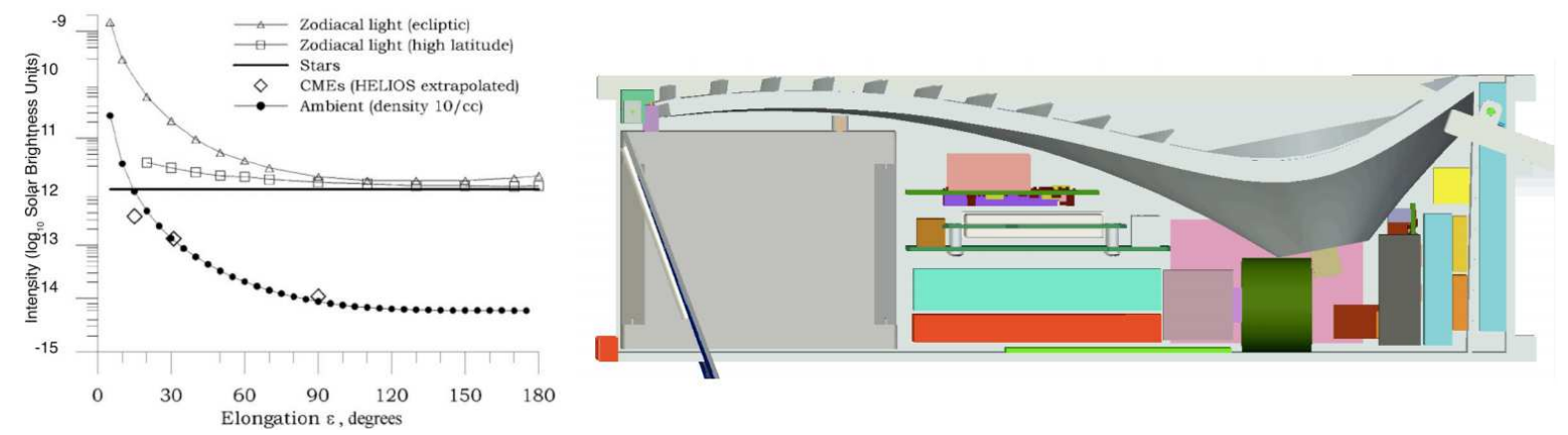

Figure 4: CHIME meets stringent stray light requirements for heliospheric imaging. LEFT: Typical ICME brightness is under $10^{-14}$ solar intensity units. The CHIME baffle and optics together reduce midfield stray light to a few $\times 10^{-15} B_{\text {sun }}$, and the CCD is sensitive to changes well under $10 \%$, giving large margin for bright ICME detection. ${ }^{4}$ RIGHT: The CHIME baffle dominates the $+Y$ face of the $3 \mathrm{U}$ CubeSat, to reduce Fresnel diffraction of solar light. 
hits, the optics are folded with an antireflective coated fused quartz prism from Meadowlark. The prism was selected for its low scatter, high transparency, and robustness against dust contamination compared to a front-coated mirror.

CHIME utilizes the compact PixelFly scientific CCD camera manufactured by Cooke. This is a 6 micron pixel, $1300 \times 1000$ device with electronic shutter and quantum efficiency of $50 \%$. The camera is operated at $0-10 \mathrm{C}$, and is cooled by direct thermal linkage between the baffle and the focal plane support structure. The readout noise is 7 e- RMS, and typical dark current is less than $20 \mathrm{e}-/ \mathrm{sec}$. The camera communicates via USB directly to the CHIME C\&DH computer, and is operated with exposure time of 3-10 seconds. Multiple camera frames are co-added in the computer, with temporal despiking to remove cosmic ray hits in individual frames.

Focusing the optics is performed on the ground using thrust setscrews and corrected for the vacuum/air difference, then the eyepiece is locked with lateral setscrews and cemented in place with vacuumcompatible epoxy. The depth of field for pixel-limited focus is 12 microns, tight but manageable in the laboratory. Over $\mathrm{a} \pm 30 \mathrm{~K}$ range of temperatures, the ideal focus position migrates by just \pm 1.2 micron due to expansion effects in the structure and optics. CHIME's temperature on-orbit is actively managed to within a few degrees. CHIME images large structures and can tolerate fixed focus shifts of up to \pm 24 microns, eliminating the need for on-orbit focus adjustment.

In normal operation the camera is electronically shuttered. We incorporate a small emergency/calibration shutter that can be used to block the main optical beam in case of pointing loss or for dark current calibration. The shutter consists of a small piece of aluminum supported by the shaft of a small
COTS bidirectional latching solenoid with $9 \mathrm{~mm}$ throw. The solenoid is identical to the rail deployment pinpullers, and is commanded by the spacecraft C\&DH computer. The shutter can be commanded closed for dark exposure calibrations, and is closed automatically if/when the ADACS leaves fine pointing mode. It is static when unpowered.

\section{Measurement Sensitivity}

The Pixelfly camera in CHIME has 6 micron pixels, quantum efficiency of 0.5 , and digitizer calibration constant close to $0.25 \mathrm{ct} / e^{-}$. Thus, in a nominal 300second macro-exposure a $10^{-14} B_{\text {sun }}$ feature (large CME) will produce over 600 digitizer counts per pixel, clearly detectable over the expected dark noise level of 30-50 counts. Taking CHIME's sensitivity to be limited by a $\mathrm{S} / \mathrm{N}$ of unity in a $4 \times 4$ pixel average, CHIME will be able to detect features as weak as 12 counts per pixel, or $2 \times 10^{-16} B_{\text {sun }}$ in the absence of stray light. Near the sunward side of the field of view, stray light and $\mathrm{F}$ corona limit the sensitivity to approximately $6 \times 10^{-16}$ $B_{\text {sun }}$ in a single 5 minute macro-exposure.

\section{SPACECRAFT SYSTEMS}

The CHIME bus is a complete, fully capable 3-axis stabilized spacecraft. Because of the high computational and data transfer requirements, CHIME is built around a highly capable C\&DH computer that supports direct communication with major subsystems via logic-level UART serial lines and with the instrument via USB. Here we describe the main blocks of spacecraft functionality.

\section{Structure and launch rails}

CHIME is built up from a basic box structure implemented in T6061 Aluminum. The $+Y$ face is replaced with the optical baffle, which is fabricated by hogging out a single block of $\mathrm{T} 6061 \mathrm{Al}$ to $6 \mathrm{~mm}$
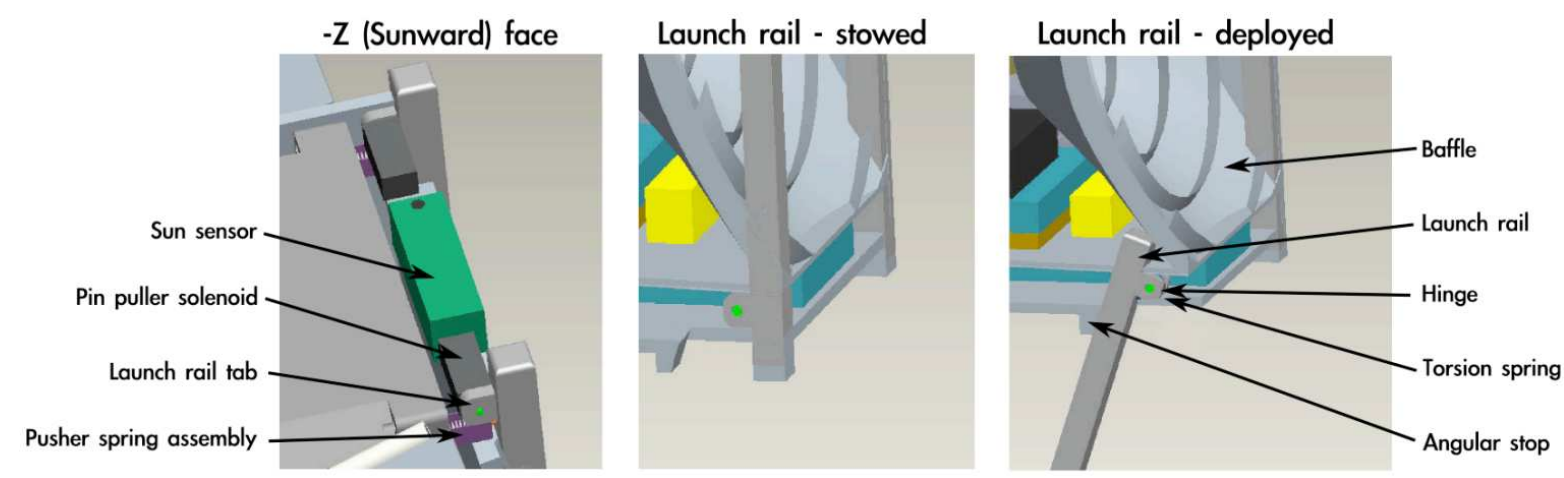

Figure 5: CHIME's baffle-side launch rails deploy behind the baffle, to prevent glint in flight. In the PPOD, the rails are secured by tab-and-pin latches on the $-Z$ end and by tab-and-pin hinges on the $+Z$ end. (embedded captive-pin separation springs in rail ends are not rendered). 
thickness. EGSE connectors and an RBF arming pin are present on the $+X$ face. The initial design is for launch loads of $20 \mathrm{G}$ (DC + RMS shake).

The launch rails on the $+Y$ side are deployable to eliminate glint into the baffle (Figure 5). The rails are secured with a tab-and-pin latch near the $-Z$ end, and a tab-and-pin hinge near the $+Z$ end. The rails deploy via pre-loaded torsion springs in the hinges. Deployment is actuated by COTS latching solenoid pin-pullers at the front of each rail. The solenoids are made by Magnetic Sensor Systems and have $9 \mathrm{~mm}$ throw, a pulling force well over $100 \mathrm{~N}$, and a latching force of $25 \mathrm{~N}$. The latch tabs seat against fixed structures in two directions, ensuring positive support. The tabs and pins are sized for inward support loads of $350 \mathrm{~N}$, compatible with a planned launch load of up to $20 \mathrm{G}$ with a factor-of-2 strength margin. An additional coil spring near the taband-pin latch provides extra deployment force in the first $3 \mathrm{~mm}$ of travel, to ensure positive action when the latch pin is pulled. CHIME's separation springs are embedded spring-and-captive-pin assemblies cemented into small bored-out holes in the ends of two rails as in the CubeSat spec. CHIME has a single deployment

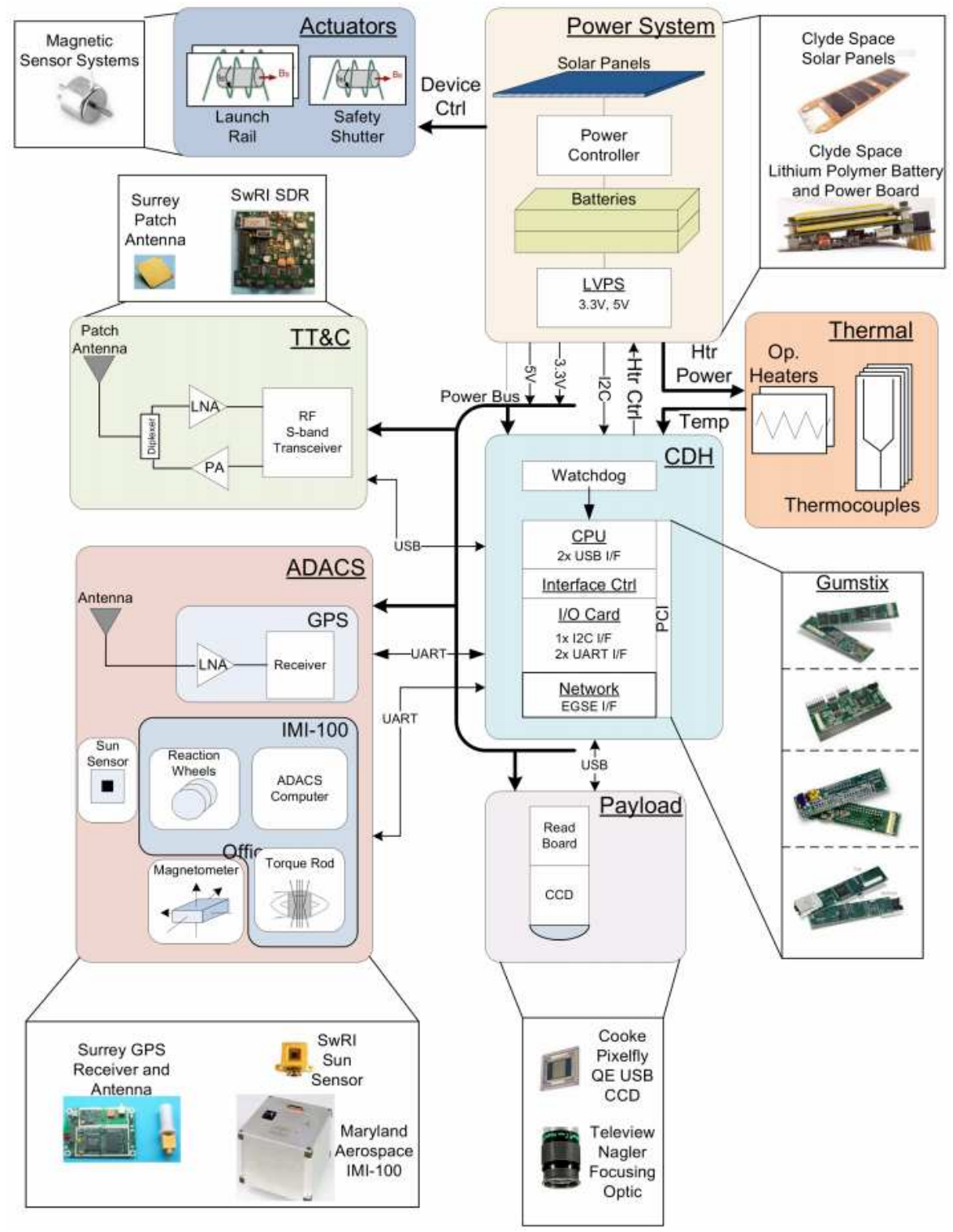

Figure 6: CHIME block diagram shows simple interfaces tied together via the COTS C\&DH computer. The only major non-COTS components - the baffle and the S-band radio - are being developed with SwRI internal funding and outside grants. 
switch on the $-\mathrm{X},-\mathrm{Y}$ rail.

\section{Attitude Determination and Control System (ADACS)}

CHIME requires arcminute class pointing relative to the Sun-Earth axis, and sub-degree pointing in the roll direction about the Sun-S/C line. We have baselined the Intellitech IMI-100, a miniature 3-axis reaction wheel ADCS designed specifically for CubeSats. The IMI-100 has one degree pointing precision with the supplied sensors and can accept analog feedback for a fine pointing mode with relative precision as fine as a few arcseconds. It is controlled via a UART serial port, and accepts inertial-frame target pointing vectors.

We augment the IMI-100 with a small solar sensor consisting of a $2 \mathrm{~mm}$ diameter lens and a commercial quad photodiode array, mounted on the front face of CHIME. The solar sensor defines the spacecraft boresight direction and is fixed before delivery. The sensor is built at COSGC and tested and integrated at SwRI. Tolerance between the solar sensor boresight and the baffle mount angle is approximately 30 arcminutes, large enough that on-orbit adjustment is not required.

\section{Control \& Data Handling}

All control and housekeeping functions are handled by a Gumstix Verdex computer running Linux. The Gumstix is a highly capable pentium-class computer and handles command and control, housekeeping, and data acquisition, compression and storage. Two separate journaling filesystems on flash RAM provide a redundant Linux bootstrap path, enabling recovery if the main filesystem is damaged. The Gumstix is located near the geometric center of the payload for maximum radiation shielding, and surrounded by an EMI enclosure to avoid interference with the GPS. An analog watchdog timer on the power board powercycles the Gumstix in case of latch-up. On-board data storage is handled with an 8GB flash card, which is partitioned and accessed via Linux kernel errorcorrecting RAID drivers as a $2.5 \mathrm{~GB}$ volume with a journaling filesystem. Each macro-exposure is $2.5 \mathrm{MB}$, enabling storage of nearly a thousand macro-exposures on board with no compression. Signal I/O is handled by a Robostix extension card, which contains several UARTs, logic level I/O lines, ADCs, and DACs.

The Linux/POSIX environment enables rapid software development. Flight functions are handled with a suite of autonomous UNIX daemons that handle pointing, telemetry, time-tagging, science events, and command parsing. The data acquisition system includes timedomain voting algorithms to eliminate cosmic ray hits. The kernel USB camera driver is supplied by Cooke and enables access to the camera via a UNIX device file rather than low level bit operations.

\section{Power}

Power is supplied by three strings of six high-efficiency solar cells supplied by ClydeSpace, and conditioned using the ClydeSpace $3 \mathrm{U}$ CubeSat power conditioner. The deployable strings supply $17 \mathrm{~W}$ with $30 \%$ margin. The power budget is given in Table 1 for three key modes: nominal data acquisition, nominal contact, and pointed dark mode. CHIME is $6 \mathrm{~W}$ positive with ample margin during normal data ops. Depending on as-built SDR performance (nominally $8 \mathrm{~W}$ ), CHIME may go slightly power negative during ground contacts, using under $5 \%$ of battery reserves during each contact. Two additional solar cells are mounted on the dark face of the -Y solar panel, ensuring that in all credible unpointed geometries, CHIME is time-average power positive in a "warm" safe mode in the nominal orbit. (In orbits with extended dark periods, a watchdog timer controls a "cold" safe mode that charges the battery, waking to "warm" mode at several-hour intervals).

The prime solar panels are slightly customized. On the $+X$ and $-X$ panels, the hinge is cut at a 20 degree angle, so that when deployed the panels angle slightly away from the baffle, eliminating glint. We have accounted for the panel geometry in the power calculations in Table 1.

\section{Tracking}

To track the satellite position, a 12-channel GPS receiver provides orbital position to within $20 \mathrm{~m}$ and synchronized GPS time. This information allows CHIME to time and date stamp telemetry records, and eliminates the need for transponder-style ranging during nominal ops (incoherent ranging is possible but not normally used). CHIME uses a Space GPS Receiver from Surrey, which includes a quadrifilar antenna to receive GPS signals and a UART to communicate with CHIME.

\section{Communications}

CHIME uses a Universal S-band software-defined radio (SDR) that is under current development with internal funding from SwRI. The full-duplex SDR transceiver has $1 \mathrm{Mbps}$ downlink bandwidth, enabling downlink of a whole orbit of solar system images within a single 6minute ground system pass, and $\sim 30 \mathrm{kbps}$ uplink bandwidth for commanding. The SDR is a 2-band transceiver and can be configured to bent-pipe mode for coherent ranging. Broadcast and reception are via ganged low-gain patch antennas on the rear $(+Z)$ and nadir (-Y) faces of the spacecraft. The SDR can receive and act on a transmitter shutdown command while transmitting. Standby power is well under $1 \mathrm{~W}$. The CHIME SDR radiates a nominal 2 Watts and we have budgeted for power consumption of 8 Watts plus an 
engineering reserve of $100 \%$ during station passes; those numbers will be refined based on actual performance once the SwRI team delivers their internally funded prototype in Summer 2010.

The nominal comms provider is United Space Network, via their Alaska ground station (or a lower latitude station for non-polar orbits). Calculated link margin is over $10 \mathrm{~dB}$ throughout each nominal 6-minute contact with the full $1 \mathrm{Mbps}$ bandwidth with USN's standard 4meter dishes. USN offers a variety of standard modulation schemes and delivers data via the internet with low enough latency to allow real-time commanding during passes. For telemetry volume, we baseline five 5-minute passes per day during CHIME's nominal mission. Communication to the radio is via a UART logic-level serial interface operated at $1 \mathrm{Mbps}$.

\section{Thermal management}

CHIME's internal temperature is actively controlled. Temperature is maintained near 30C using a combination of multilayer insulation, variable albedo (the normally sunlit $-\mathrm{Z}$ face is painted white; the normally dark faces are painted black to absorb sunlight in "tumbling" safe mode), and thermostatically controlled heating elements. The $6 \mathrm{~W}$ heaters are controlled by the C\&DH computer based on thermal data from four thermistors located around CHIME, and can adjust CHIME internal temperature between OC and 45C. The camera and optics are thermally isolated from the surrounding structure and coupled directly to the baffle, which is exposed to deep space; initial estimates of operating temperature are close to $0 \mathrm{C}$, compatible with desired temperature range for best CCD performance.

\section{De-Orbit}

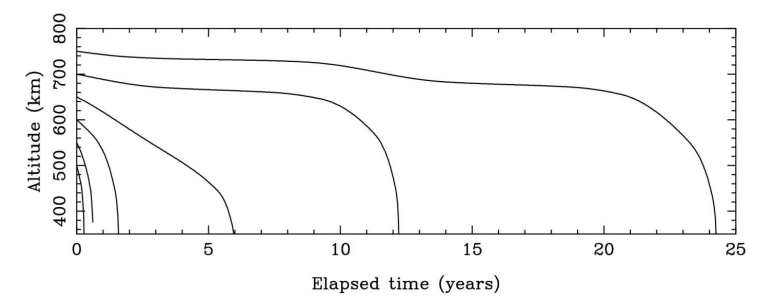

Figure 7: CHIME de-orbits in under 25 years from near-circular altitudes up to $750 \mathrm{~km}$ due to its large deployable structures and low mass. These dynamic simulations assume circular orbit and take solar cycle effects into account as a perturbation on the Harris-Preister model atmosphere. ${ }^{10}$ CHIME is launched in 2013, close to solar maximum.
CHIME de-orbits passively in under 25 years from the nominal $750 \mathrm{~km}$ altitude circular orbit (Figure 7) due to its low mass and large deployable structures. Higher orbits require additional drag mechanisms or a variance on decay time requirements.

\section{COMMANDING}

CHIME contacts commence when the SDR identifies a carrier signal query from the ground and establishes two-way communications. CHIME responds with a handshake challenge query that is implemented using the standard /bin/login code. Upon successful handshake, CHIME enters a simple command shell that allows uplink of batch command files and downlink of stored telemetry and HK files via command sent from the ground system.

Normally, commands are uplinked as new configuration files or time-tagged command tables in the UNIX environment aboard CHIME, and are transferred and verified in the same way as downlinked data files. Uploaded files that fail the md5 checksum generate an error message in the HK telemetry and are resent by the ground system until a successful md5 checksum is achieved. Because the radio is treated as a standard tty device by the UNIX device drivers, CHIME can also be commanded interactively via direct login to the UNIX shell. This is a useful mode for pre-delivery debugging, and is left in-place during the flight as a disaster recovery mode.

\section{Data transfer \& compression}

CHIME transfers data as an unformatted stream of 8-bit bytes via UART to the $\mathrm{S}$-band radio, greatly simplifying data transfer. Error correction coding is accomplished at the stream level and occurs on-the-fly within the CPU. Files are transferred from CHIME using a standard stream transfer protocol such as rsync or kermit, with an additional high-level wrapper to verify file integrity. Each data file transferred up or down is matched with a separate md5 checksum file with related file name, and the receiver calculates the checksum on the ground before issuing a final ACK or NAK command to the sender.

CHIME housekeeping data are maintained on board as a collection of human-readable ASCII files that are compressd with "gzip" Huffman coding before downlink. CHIME image data are maintained on board as FITS files. Losslessly compressed files use the existing FITS standard for tile compression with Rice compression; lossily compressed files are waveletcompressed with a variant of JPEG2000 that was developed at SwRI and optimized for astronomical images as part of a previous project. The wavelet- 
compressed stream is stored as an entry in a one-row FITS binary table.

\section{MISSION OPERATIONS \& GROUND SYSTEM}

CHIME operations are conducted via the Internet from SwRI and/or COSGC, using computers located at SwRI. The baseline operations plan utilizes 4 station passes per day, enabling transfer of 290 2MB macroexposures per day with a near-lossless image compression ratio of 4.0, sufficient for sustained CHIME operation at 5 minute macro-exposure cadence. (Future missions will utilize more passes per day to reduce data latency.)

A single command-and-control computer, located at SwRI, receives telemetry data from USN and generates and transmits command loads. A data reduction computer receives files from the command computer, reduces them to usable form, and serves them to the Internet via the WWW. Housekeeping data are automatically plotted and placed on the project web page for easy browsing; raw science data are uncompressed and placed online as FITS images, also on the project web page. Because the CHIME housekeeping data are produced in a simple format (human-readable ASCII tables) and have few individual data tracks, they are easily parsed and plotted. CHIME images are delivered as FITS files and require only minimal processing to be scientifically useful. Both original and uncompressed FITS files are available on the web server.

Typical passes are unattended and have no commanding or configuration changes to the spacecraft. The ground system autonomously logs in to CHIME with the downlink login, and CHIME immediately starts a file dump operation. The data are transferred over the Internet from the USN ground system to SwRI's commanding computer and unpacked there.

\section{DATA DISTRIBUTION}

To encourage scientific collaboration, All CHIME data will be placed on a publicly accessible web server as soon as practical after it becomes available to the CHIME team (typically within 1 day of receipt). Calibration information will be placed on the same web server as it becomes available. Provisional calibrations and background subtracted data will be made available as soon as possible. A list of student science projects will be posted, and non-team scientists will be asked to avoid using CHIME data for those topics for a waiting period, to allow the students time to work.

\section{Software Components}

Because the main CHIME flight computer uses high capability commercial parts and runs Linux, development costs are low. Standard software development tools are used on-board. Noncritical functions are handled in a high level data reduction environment such as Numeric Python or Perl Data Language. Preemptive multitasking in the POSIX environment reduces complexity of the software components. On-board nonvolatile RAM is organized with a standard journaling file system. The CPU watchdog system is maintained with a background daemon. Individual software components are watched by a "watchdog daemon" that looks for semaphores in a volatile RAM filesystem.

\section{Flight Control Software}

CHIME flight control software is an autonomous highpriority UNIX daemon that is started automatically on boot. The boot sequence uses a checksummed kernel image that unpacks to a RAM disk. This standard standard microcontroller boot procedure allows smooth fail-over to a redundant boot image.

\section{On-board data handling}

CHIME data handling requires several internal components that are closer to a ground-based data pipeline than a typical flight acquisition system. Image data are acquired from the camera via a high speed serial link, and then handled within the POSIX system. Raw images from the camera are conditioned, examined, prioritized for downlink, and compressed. The downlink queue is stored as a directory in a Flash RAM filesystem, and files are downlinked using a simple streaming protocol with AES encryption.

\section{Image acquisition, Processing, \& Selection}

CHIME uses multi-image acquisition to minimize the effects of particle hits to the CCD during exposure. Rather than a single minutes-long exposure CHIME acquires $10-30$ or 10-second exposures in succession, and uses a time based scheme ("ZSPIKE") on-board to identify and remove particle hits from the image, before further processing or downlink occurs. ZSPIKE has been tested on actual data from the TRACE solar imaging spacecraft. TRACE data are severely degraded during passage through the polar caps or SAA. ZSPIKE removes spikes and delivers "clean" data from 10 -image sequences even when more than $2 \%$ of pixels in each image are degraded by cosmic ray "hits" - this corresponds to approximately $18 \%$ obscuration of the data by cosmic ray hits. ZSPIKE preserves the starfield. 
After acquisition and despiking, "macro-exposures" are tested for large bright regions that indicate the Earth or Moon are in the field of view and/or contributing stray light. This step yields summary heuristics that are used to prioritize image downlinks during passes. Finally, each image is lossily compressed and stored in the flash RAM file system to await downlink. During each pass, the highest priority image is downlinked first. Priority for each image shrinks as time passes, so that fresh high quality images have highest priority for downlink, and old images are deleted when their priority drops sufficiently low.

\section{EXPECTED PERFORMANCE}

A single CHIME CubeSat will yield heliospheric image data at comparable cadence to the existing SMEI imager on board the Coriolis spacecraft, enabling prediction and diagnosis of the effect of space weather events well before they impact Earth's magnetosphere. CHIME images are expected to be higher quality than SMEI images because CHIME utilizes a full twodimensional imaging system and on-board cosmic ray detection and removal, while SMEI utilizes narrow, nearly 1-D image strips. Two-dimensional imaging allows CHIME to record a particular piece of the celestial sphere multiple times during an orbit, from locations separated by up to $6000 \mathrm{~km}$. This eliminates both major sources of noise in the SMEI data: high altitude geocorona under which the spacecraft passes during its orbit, and particle showers during passage through the polar caps and SAA.

CHIME spatial resolution is 7-10 arcminutes (3.5 arcmin pixels), comparable to SMEI and sufficient to resolve and remove bright stars. Elongation range is 35 - 95 degrees, allowing tracking of Earth-directed ICMEs from about $0.5 \mathrm{AU}$ to impact. Image acquisition time is under 300 seconds for a single 60 degree diameter macro-exposure; several such exposures are patched together on the ground to yield sky maps of the inner solar system. The CHIME constellation yields approximately one sky map per orbital period, per active spacecraft. Predictive capability has been demonstrated using 90 minute sky map cadence (with SMEI data), but improves with more rapid cadence.

The most critical performance parameter for the CHIME instrument is brightness detection threshold, which is a combination of stray light performance and detector discrimination capability. With a 300 second macro-exposure, CHIME's estimated sensitivity is $10^{-16} \mathrm{IS}$, giving two orders of magnitude of margin compared to the requirement of $10^{-14}$ IS.
For operational capability, data volume and latency are of critical importance. Using the baseline S-band transceiver with $1 \mathrm{Mbps}$ bandwidth, data volume is 20 $30 \mathrm{MB}$ per four-minute pass, enabling downlink of up to 30 macro-exposures per pass with 2:1 near-lossless compression. In this mode, all data acquired by CHIME can be downlinked using 2-4 ground stations depending on placement, yielding highest image quality and under 1 hour latency from data acquisition to distribution of calibrated data from the ground system server. Using the demonstration UHF transceiver with 9600 bps bandwidth, data volume is $280 \mathrm{~KB}$ per fourminute pass. In this mode, images are compressed 25:1 before downlink, and 2-3 compressed macro-exposures may be downlinked during each pass. The wavelet compression method used by CHIME has been tested against real coronagraph and all-sky imaging data and shown to reproduce ICME location well enough for leading-edge detection. ${ }^{11}$ In this mode, ground station count greatly affects total data volume and prediction quality.

\section{References}

1. Baker, D.N., Balstad, R., Bodeau, J.M., et al., "Severe Space Weather Events: Understanding Societal and Economic Impacts," The National Academies Press, Washington, DC, May, 2009.

2. Yashiro, S., Gopalswamy, N., Michalek, G., et al., "A Catalog of White Light Coronal Mass Ejections Observed by the SOHO Spacecraft," Journal of Geophysical Research, Volume 109, July, 2004.

3. Richter, I., Leinert, C., Planc, B., "Search for Short Term Variations of Zodiacal Light and Optical Detection of Interplanetary Plasma Clouds," Astronomy and Astrophysics, Volume 110, pg. 115, March, 1982.

4. Eyles, C.J., Simnett, G.M., Cooke, M.P., et al., "The Solar Mass Ejection Imager (SMEI)," Solar Phys. 217, 319, 2003.

5. Howard, T.A., Tappin, S.J., "Interplanetary Coronal Mass Ejections Observed in the Heliosphere: 3. Physical Implications," Space Sci. Rev. 147, 89, 2010.

6. Webb, D.F., Howard, T.A., Fry, C.D., et al., "Studying Geoeffective Interplanetary Coronal Mass Ejections Between the Sun and Earth: Space Weather Implications of Solar Mass Ejection Imager Observations," Space Weather 7, 2009.

7. Smith, Z.K., Dryer, M., "The Interplanetary Shock Propagation Model: A model for ... Global 
Model (2D IGM)," NOAA Technical Memorandum, ERL/SEL 89, 1995.

8. Howard, T.A., Tappin, S.J., "The Application of a New Phenomenological Coronal Mass Ejection Model to Space Weather Forecasting," Space Weather, in press, 2010.

9. Tappin, S.J., Howard, T.A., "Interplanetary Coronal Mass Ejections Observed in the Heliosphere: 2. Model and Data Comparison," Space Sci. Rev. 147, 55, 2009.

10. Vallado, D.A., Finkleman, D., "A Critical Assessment of Satellite Drag and Atmospheric Density Modeling," AIAA/AAS Astrodynamics Specialist Conference and Exhibit, August, 2008.

11. DeForest, C.E., et al., "DAMASC: A Compact Coronagraph for Space Weather Applications," project final report, NOAA/SWPC, 2007. 